\title{
CORRELATION BETWEEN INTRASAC PRESSURE MEASUREMENTS OF A PRESSURE SENSOR AND AN ANGIOGRAPHIC CATHETER DURING ENDOVASCULAR REPAIR OF ABDOMINAL AORTIC ANEURYSM
}

\author{
Pierre Galvagni Silveira, Christopher William Teixeira Miller, Rafael Freygang \\ Mendes, Gilberto Nascimento Galego
}

\begin{abstract}
Silveira PG, Miller CWT, Mendes RF, Galego GN. Correlation between intrasac pressure measurements of a pressure sensor and an angiographic catheter during endovascular repair of abdominal aortic aneurysm. Clinics. 2008;63(1):59-66.
\end{abstract}

PURPOSE: To establish a correlation between intrasac pressure measurements of a pressure sensor and an angiographic catheter placed in the same aneurysm sac before and after its exclusion by an endoprosthesis.

METHODS: Patients who underwent endovascular abdominal aortic aneurysm repair and received an EndoSure ${ }^{\mathrm{TM}}$ wireless pressure sensor implant between March 19 and December 11, 2004 were enrolled in the study. Simultaneous readings of systolic, diastolic, mean, and pulse pressure within the aneurysm sac were obtained from the catheter and the sensor, both before and after sac exclusion by the endoprosthesis (Readings 1 and 2, respectively). Intrasac pressure measurements were compared using Pearson's correlation and Student's t test. Statistical significance was set at $\mathrm{p}<0.05$.

RESULTS: Twenty-five patients had the pressure sensor implanted, with simultaneous readings (i.e., recorded by both devices) obtained in 19 patients for Reading 1 and in 10 patients for Reading 2. There was a statistically significant correlation for all pressure variables during both readings, with $\mathrm{p}<0.01$ for all except the pulse pressure in Reading $1(\mathrm{p}<0.05)$. Statistical significance of pressure variations before and after abdominal aortic aneurysm exclusion was coincident between the sensor and catheter for diastolic $(\mathrm{p}>0.05)$, mean $(\mathrm{p}>0.05)$, and pulse $(\mathrm{p}<0.01)$ pressures; the sole disagreement was observed for systolic pressure, which varied, on average, $31.23 \mathrm{mmHg}$ by the catheter $(\mathrm{p}<0.05)$ and $22 \mathrm{mmHg}(\mathrm{p}>0.05)$ by the sensor.

CONCLUSION: The excellent agreement between intrasac pressure readings recorded by the catheter and the sensor justifies use of the latter for detection of post-exclusion abdominal aortic aneurysm pressurization.

KEYWORDS: Post-exclusion. Endoleak. Endoprosthesis. Pressure measurements. Aneurysm sac

\section{INTRODUCTION}

Endovascular aneurysm repair may be applicable for up to two-thirds of abdominal aortic aneurysms (AAA). However, although the results of this approach exceed those of conventional therapy in a great number of patients, the risk of rupture after endoluminal repair remains relatively high (approximately $1 \%$ per year). ${ }^{1}$

General Surgery, Federal University of Santa Catarina - Florianópolis/SC cwtmiller@yahoo.com.br

Received for publication on October 11, 2007.

Accepted for publication on November 07, 2007.
Among the most common complications of endovascular aneurysm repair with placement of endoprostheses are endoleak, an ongoing perfusion and pressurization of the aneurysm sac after its supposed exclusion, ${ }^{2}$ which affects 8 to $44 \%$ of AAA treated with endoprosthesis, ${ }^{3}$ and endotension, an aneurysm enlargement without a visible endoleak. ${ }^{4}$

Imaging methods are currently advocated as the firstchoice examination for the assessment of postexclusion AAA pressurization ${ }^{5}$ even though these techniques have inherent limitations that make them inaccurate for detecting certain endoleaks. ${ }^{6}$ 
Direct intrasac pressure measurement is by far superior to imaging techniques in ascertaining either the complete AAA exclusion or the need for future interventions. ${ }^{7}$ Intrasac pressure can be measured intraoperatively by a catheter delivered through femoral access into the AAA sac. During postoperative follow-up, the main resource for pressure measurement is translumbar percutaneous puncture, ${ }^{4,7,8}$ which is an invasive procedure requiring fluoroscope guidance for precise positioning of the needle and involving radiation exposure and use of contrast medium. In addition, there is an associated risk of infection and endoprosthesis perforation, the latter limiting its use in cases of great proximity between the vessel wall and the endoprosthesis. ${ }^{8,9}$

Ellozy et al. reported the first study in humans to utilize an implantable, ultrasound-activated remote pressure transducer to measure intrasac pressure after endovascular aneurysm repair. ${ }^{10}$ CardioMEMS, Inc. has developed another type of technology for the same purpose. Their biocompatible sensor is activated by radiofrequency. An external source records the sensor's resonant frequency which, in turn, varies according to the pressure at the sensor's location.

It is of particular importance to investigate whether noninvasive pressure sensors are as accurate as invasive methods for intra-aneurysm sac pressure measurement and, if so, whether they could be used as the only source for monitoring intrasac pressure during postoperative follow-up.

\section{MATERIAL AND METHODS}

\section{Study Design}

Analysis of intraoperative data for assessment of correlation.

\section{Data Source}

This study was designed to analyze intraoperative data from patients with infrarenal AAA treated by an endovascular approach at the hemodynamics room of Hospital Universitário, at the SOS Cardio Clinic and at the Hospital de Caridade (all located in the city of Florianópolis, SC, Brazil), between March 19 and December 11, 2004. The aforementioned patients took part in a clinical trial that was sponsored by CardioMEMS Inc. ${ }^{11}$ and approved by the Ethics in Research Committee on March 19, 2004. All patients signed an informed consent form.

\section{Inclusion Criteria}

Male or female patients at least 18 years of age undergoing endovascular therapy for treatment of abdominal aortic aneurysms and compliant with protocol requirements were enrolled in this study. Anatomic criteria included having adequate space (more than $10 \mathrm{~mm}$ ) between the aneurysm wall and the endoprosthesis for implantation of the sensor.

\section{Device Description}

The EndoSure ${ }^{\mathrm{TM}}$ wireless pressure sensor (Figure 1) measures approximately $30 \times 35 \mathrm{~mm}$. It is constructed by laminating together several layers to form a capacitor. Metal spirals in the first and last layer form the inductor components of an electrical circuit. Current induction in the sensor results in energy oscillation that varies with frequency. Changes in the circuit's resonant frequency are directly proportional to the force applied to the sensor's surface (in this case, the pressure within the aneurysm sac).

The inductor allows electromagnetic coupling between the sensor and the electronic system. The latter consists of an antenna held against the patient's side or back in the area where the sensor is located; it measures the resonant frequency, which is then displayed on a computer screen (Figure 2).

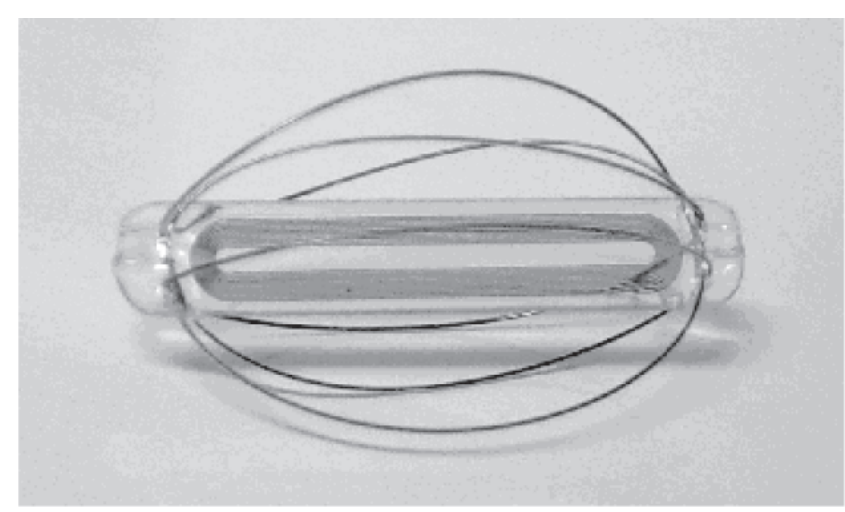

Figure 1 - EndoSure ${ }^{\mathrm{TM}}$ wireless pressure sensor.

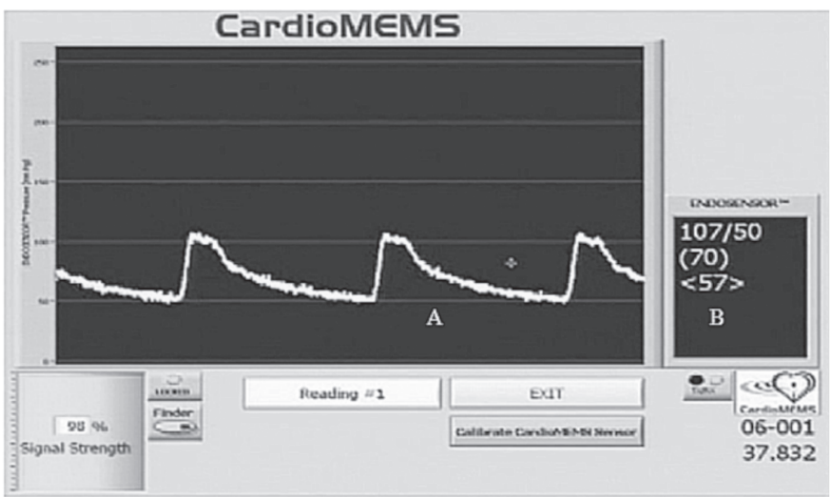

Figure 2 - Pressure wave and levels measured by the sensor during Reading 1. A. Pressure wave within the aneurysm sac. B. Various pressure levels within the sac. From top to bottom: systolic/diastolic, mean, and pulse pressure. 


\section{Description of the Technique}

Intrasac pressure measurements were performed at two time points. After delivery and deployment of the main body of the endoprosthesis, both the angiographic catheter and the sensor performed Reading 1 (Figure 3), which consisted of recording the systolic, diastolic, mean, and pulse pressures. Once the contralateral extension of the endoprosthesis was introduced and the sensor was released from the guidewire, the angiographic catheter (if not removed after Reading 1) and the sensor performed new pressure measurements after aneurysm exclusion from systemic circulation (Reading 2) (Figure 4). If no endoleak was detected by fluoroscopy, the catheter was removed and the procedure was considered completed.

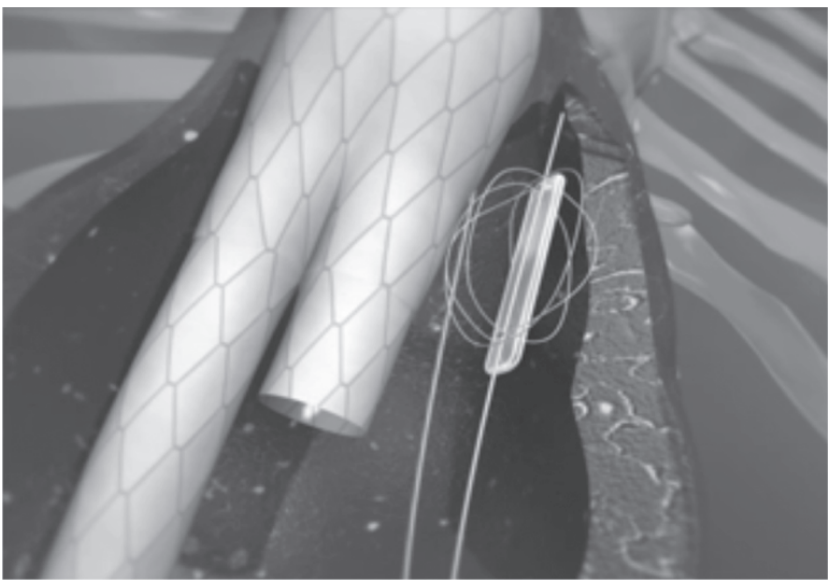

Figure 3 - The technique employed for determining intra-sac pressure, Reading 1. Placed within the sac are the angiographic catheter and, nearby, the pressure sensor

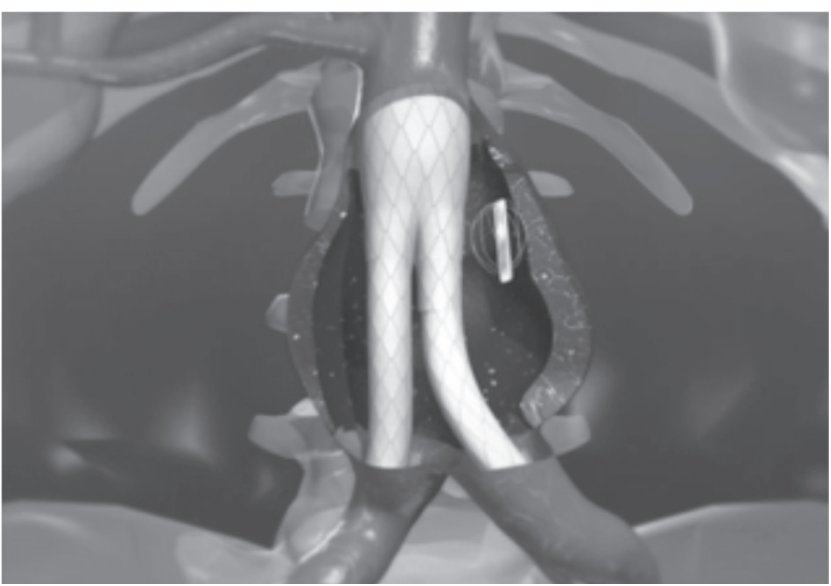

Figure 4 - Visualization of the EndoSure ${ }^{\mathrm{TM}}$ wireless pressure sensor after sac exclusion by the endoprosthesis.

\section{Statistical Analysis}

Systolic, diastolic, pulse, and mean pressures from Reading 1 and Reading 2 were analyzed on the following bases: (1) to define agreement between simultaneous intrasac pressure measurements recorded by the angiographic catheter and the pressure sensor, using Pearson's correlation; and (2) to define whether there was a statistically significant difference between the angiographic catheter measurements (in the absence of aneurysm sac pressurization) and whether the pressure sensor readings were consistent with these findings. The Student's paired t-test was used for this analysis.

In both cases, the results were considered statistically significant at the level $p<0.05$. Statistical analysis and scatter plot design were performed using Microsoft ${ }^{\circledR}$ Excel 2002.

\section{RESULTS}

\section{Data Source Characterization}

Twenty-five patients had the pressure sensor implanted together with the endoprosthesis, but only 19 patients had their intraoperative data analyzed. In four patients, no angiographic catheter intraoperative pressure readings were collected because of modifications introduced in the protocol supplied by the study sponsor (see Discussion). Two other patients were excluded from the trial, one as a result of sensor malfunction and another due to death in the postoperative period as a result of decompensated congestive heart failure (death occurring in the immediate postoperative period due to a condition unrelated to sensor implantation was an exclusion criterion for the study). Demographic characteristics of the included patients are summarized in Table 1.

The 23 patients included in the study all received modular aortobiiliac endoprostheses, 22 supplied by $\mathrm{Nano}^{\circledR}$ and one by Talent ${ }^{\circledR}$ Medtronic. All patients had the EndoSure ${ }^{\mathrm{TM}}$ wireless pressure sensor implanted along with the endoprosthesis. The study population was composed of 19 men and four women ranging in age from 48 to 84 years, with an average age of 68.33 years. The maximum aneurysm diameter ranged from 41 to $70 \mathrm{~mm}$ (mean: 51.78 $\mathrm{mm})$.

The following results describe findings from only those 19 patients for whom statistical analysis was carried out.

\section{Intraoperative Results}

In 16 patients, there was complete exclusion of the AAA from systemic circulation, with no evidence of endoleak. One patient had a type I endoleak that was treated with ballooning of the proximal neck. Two patients had type III endoleaks; one of them received a distal extension and the 
Table 1 - Patient demographics in this study.

\begin{tabular}{lccc}
\hline Patient \# & Sex & Age & Maximum aneurysm diameter $(\mathrm{mm})$ \\
\hline 1 & Male & 58 & 44 \\
2 & Male & 79 & 44 \\
3 & Male & 63 & 70 \\
4 & Male & 67 & 45 \\
5 & Male & 64 & 53 \\
6 & Female & 57 & 45 \\
7 & Male & 73 & 45 \\
8 & Male & 48 & 42 \\
9 & Male & 72 & 50 \\
10 & Male & 65 & 41 \\
11 & Female & 81 & 59 \\
12 & Male & 70 & 64 \\
13 & Male & 53 & 45 \\
14 & Male & 68 & 45 \\
15 & Male & 60 & 45 \\
16 & Male & 71 & 62 \\
$17 *$ & Male & 73 & 54 \\
18 & Female & 73 & 70 \\
19 & Male & 64 & 57 \\
$20 \ddagger$ & Male & 64 & 61 \\
$21 \ddagger$ & Female & 73 & 48 \\
$22 \ddagger$ & Male & 81 & 47 \\
$23 \ddagger$ & Male & 66 & 49 \\
\hline
\end{tabular}

* All patients underwent AAA endovascular repair. The procedure was performed using the $\mathrm{Nano}^{\circledast}$ Apolo endoprosthesis for all patients except \#17, for whom the Talent ${ }^{\circledR}$ Medtronic device was utilized.

$\$$ Patients not included in statistical analysis (refer to text).

Note: the two patients excluded from the study are not represented in this table.

SOURCE: CardioMEMS Inc., data bank, 2004. other underwent a watchful waiting approach after administration of protamine. In neither of the cases did the endoleaks resolve during the surgical intervention; an expectant management of the patients was adopted.

The pressure sensor functioned properly in all 19 patients, which made possible the registration of all Reading 1 simultaneous intrasac measurements. Due to the abovementioned protocol modifications, nine patients (including both patients with type III endoleaks) did not have their angiographic catheter measurements recorded in Reading 2. Therefore, in Reading 2, there were simultaneous pressure measurements (i.e., by the sensor and the catheter) in only 10 patients. One of the patients in whom Reading 2 measurements were performed had a type I endoleak after endoprosthesis implantation.

\section{Reading 1 Simultaneous Intrasac Pressure Measurements}

Intrasac pressure values recorded by the pressure sensor and the angiographic catheter in Reading 1 are shown in Table 2. Pearson's correlation coefficients for systolic, diastolic, pulse, and mean pressure measurements were 0.85 $(\mathrm{p}<0.01), 0.93(\mathrm{p}<0.01), 0.50(\mathrm{p}<0.05)$, and $0.96(\mathrm{p}<0.01)$, respectively (Figure 5).

Table 2 - Intrasac pressure measurements for Reading 1 ( $\mathrm{mmHg}$ ).

\begin{tabular}{|c|c|c|c|c|c|c|c|c|}
\hline \multirow[t]{2}{*}{ Patient \# } & \multicolumn{2}{|c|}{ Systolic pressure } & \multicolumn{2}{|c|}{ Diastolic pressure } & \multicolumn{2}{|c|}{ Pulse pressure } & \multicolumn{2}{|c|}{ Mean pressure } \\
\hline & Sensor & Catheter & Sensor & Catheter & Sensor & Catheter & Sensor & Catheter \\
\hline 1 & 139 & 137 & 64 & 66 & 75 & 71 & 95 & 94 \\
\hline 2 & 107 & 111 & 61 & 58 & 46 & 53 & 79 & 78 \\
\hline 3 & 140 & 145 & 82 & 78 & 58 & 66 & 104 & 104 \\
\hline 4 & 124 & 128 & 63 & 63 & 61 & 65 & 88 & 88 \\
\hline 5 & 83 & 84 & 42 & 42 & 41 & 42 & 56 & 56 \\
\hline 6 & 92 & 102 & 64 & 57 & 28 & 45 & 75 & 74 \\
\hline 7 & 92 & 103 & 56 & 54 & 36 & 49 & 69 & 69 \\
\hline 8 & 113 & 117 & 65 & 62 & 48 & 55 & 85 & 85 \\
\hline 9 & 115 & 132 & 53 & 43 & 62 & 88 & 76 & 68 \\
\hline 10 & 159 & 178 & 94 & 82 & 65 & 97 & 118 & 117 \\
\hline 11 & 122 & 119 & 69 & 62 & 53 & 57 & 91 & 86 \\
\hline 12 & 114 & 111 & 75 & 72 & 39 & 40 & 89 & 89 \\
\hline 13 & 123 & 127 & 65 & 61 & 58 & 66 & 89 & 86 \\
\hline 14 & 107 & 117 & 54 & 55 & 53 & 62 & 74 & 78 \\
\hline 15 & 88 & 119 & 45 & 38 & 43 & 81 & 58 & 62 \\
\hline 16 & 128 & 138 & 64 & 70 & 64 & 68 & 90 & 100 \\
\hline 17 & 123 & 166 & 113 & 97 & 9 & 69 & 119 & 119 \\
\hline 18 & 119 & 125 & 64 & 57 & 55 & 68 & 85 & 86 \\
\hline 19 & 132 & 150 & 60 & 70 & 72 & 80 & 86 & 100 \\
\hline $20 *$ & 105 & - & 58 & - & 47 & - & 74 & - \\
\hline $21 *$ & 114 & - & 51 & - & 63 & - & 72 & - \\
\hline $22 *$ & 84 & - & 72 & - & 12 & - & 76 & - \\
\hline $23 *$ & 157 & - & 128 & - & 29 & - & 138 & - \\
\hline
\end{tabular}

* Patient did not have angiographic catheter readings performed.

SOURCE: CardioMEMS Inc., data bank, 2004. 

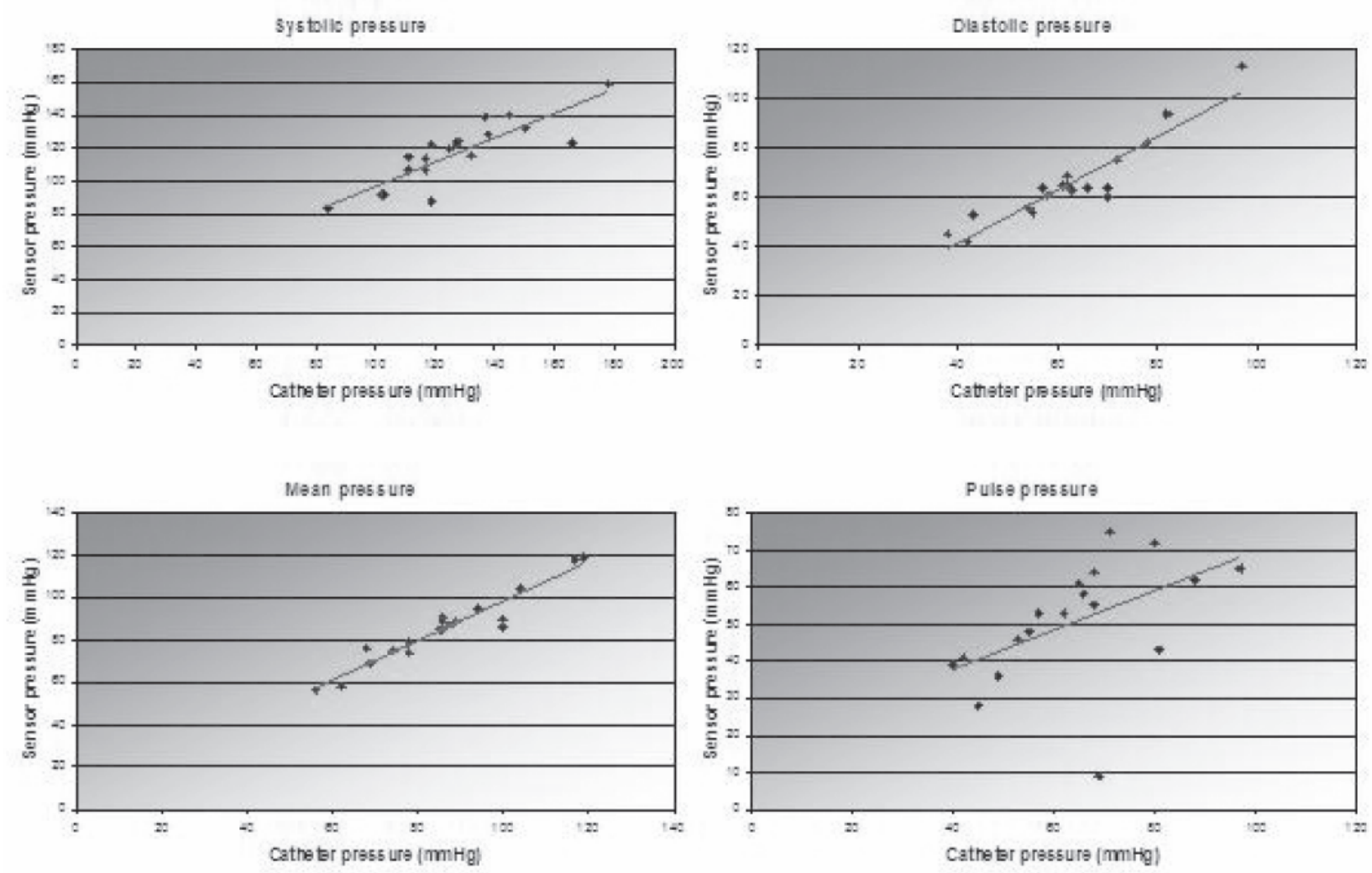

Figure 5 - Scatterplots showing agreement between the Reading 1 pressure measurements of angiographic catheter and pressure sensor.

\section{Reading 2 Simultaneous Intrasac Pressure Measurements}

Intrasac pressure values recorded by the pressure sensor and the angiographic catheter for Reading 2 are shown in Table 3. Pearson's correlation coefficients for systolic, diastolic, pulse, and mean pressure measurements were 0.87 $(\mathrm{p}<0.01), 0.75(\mathrm{p}<0.01), 0.82(\mathrm{p}<0.01)$, and $0.84(\mathrm{p}<0.01)$, respectively (Figure 6).

\section{Intrasac Pressure Variation Before and After Aneurysm Exclusion}

The pressure values recorded for patients who did not present endoleaks are given in Table 4. One patient showed type I endoleak after endoprosthesis implantation; this endoleak was detected by both the catheter and the sensor inasmuch as the pressure levels were similar to baseline.

\section{DISCUSSION}

The "gold standard" for AAA pressure measurements is the use of intrasac catheters (with a sensor tip or fluid column). ${ }^{7,12,13}$ Nevertheless, a catheter with a fluid column for pressure gauging has limited accuracy, and reading er- rors may arise from use of an excessively long catheter or an incomplete fluid column due to bubble or thrombus formation. ${ }^{7,9,10}$ These shortcomings highlight the importance of non-invasive pressure measurement methods.

In the conducted study, the angiographic catheter and the pressure sensor demonstrated what has classically been described after AAA exclusion: a decrease in levels of systolic, mean, and pulse pressures within the aneurysm sac, as well as an increase in diastolic pressure (supposedly attributed to absence of decompression of the excluded sac in diastole). ${ }^{10}$ Although the statistical significance of pressure variations has limited implications, it is important to emphasize that both pressure gauge devices detected a drop in pulse pressure, which denotes the decrease of systolic and increase of diastolic pressures. Moreover, a drop in pulse pressure was observed in all patients with the exception of the patient in whom an endoleak was detected, but this finding was expected due to continuous intrasac pressurization.

A strong correlation was found between the angiographic catheter and the pressure sensor for almost all pressure measurements of Reading 1 and Reading 2, except for the Reading 1 pulse pressure, which had a relatively poorer correlation (although still statistically significant). This poorer correlation was in great part attrib- 
Table 3 - Intrasac pressure measurements for Reading 2 (mmHg).

\begin{tabular}{|c|c|c|c|c|c|c|c|c|}
\hline \multirow[t]{2}{*}{ Patient \# } & \multicolumn{2}{|c|}{ Systolic pressure } & \multicolumn{2}{|c|}{ Diastolic pressure } & \multicolumn{2}{|c|}{ Pulse pressure } & \multicolumn{2}{|c|}{ Mean pressure } \\
\hline & Sensor & Catheter & Sensor & Catheter & Sensor & Catheter & Sensor & Catheter \\
\hline 1 & 120 & 100 & 82 & 64 & 38 & 36 & 98 & 80 \\
\hline $2 \ddagger$ & 107 & 117 & 59 & 55 & 48 & 62 & 77 & 81 \\
\hline 3 & 89 & 97 & 75 & 88 & 14 & 10 & 82 & 93 \\
\hline 4 & 90 & 91 & 70 & 55 & 20 & 35 & 80 & 71 \\
\hline 5 & 123 & 109 & 87 & 74 & 36 & 35 & 105 & 92 \\
\hline 6 & 85 & 97 & 63 & 55 & 22 & 42 & 72 & 71 \\
\hline 7 & 89 & 79 & 72 & 56 & 17 & 23 & 80 & 65 \\
\hline 8 & 81 & 74 & 62 & 55 & 19 & 19 & 72 & 65 \\
\hline 9 & 47 & 45 & 32 & 39 & 15 & 5 & 40 & 42 \\
\hline $10^{*}$ & 90 & - & 79 & - & 11 & - & 83 & - \\
\hline $11^{*}$ & 118 & - & 77 & - & 41 & - & 91 & - \\
\hline 12 & 90 & 86 & 72 & 60 & 18 & 27 & 80 & 70 \\
\hline $13^{*}$ & 130 & - & 80 & - & 50 & - & 97 & - \\
\hline $14^{*}$ & 66 & - & 53 & - & 13 & - & 57 & - \\
\hline $15^{*}$ & 83 & - & 47 & - & 36 & - & 59 & - \\
\hline $16^{*}$ & 106 & - & 66 & - & 40 & - & 79 & - \\
\hline $17 *$ & 117 & - & 108 & - & 9 & - & 111 & - \\
\hline $18^{*}$ & 141 & - & 72 & - & 69 & - & 95 & - \\
\hline $19 *$ & 124 & - & 71 & - & 53 & - & 89 & - \\
\hline $20^{*}$ & 100 & - & 70 & - & 30 & - & 80 & - \\
\hline $21^{*}$ & 80 & - & 56 & - & 24 & - & 64 & - \\
\hline $22^{*}$ & 84 & - & 47 & - & 37 & - & 59 & - \\
\hline $23^{*}$ & 128 & - & 113 & - & 15 & - & 118 & - \\
\hline
\end{tabular}

\$ Patient 2 presented a type I endoleak after insertion of the contralateral extension of the endoprosthesis.

* Patients did not have angiographic catheter measurements performed for Reading 2.

SOURCE: CardioMEMS Inc., data bank, 2004.
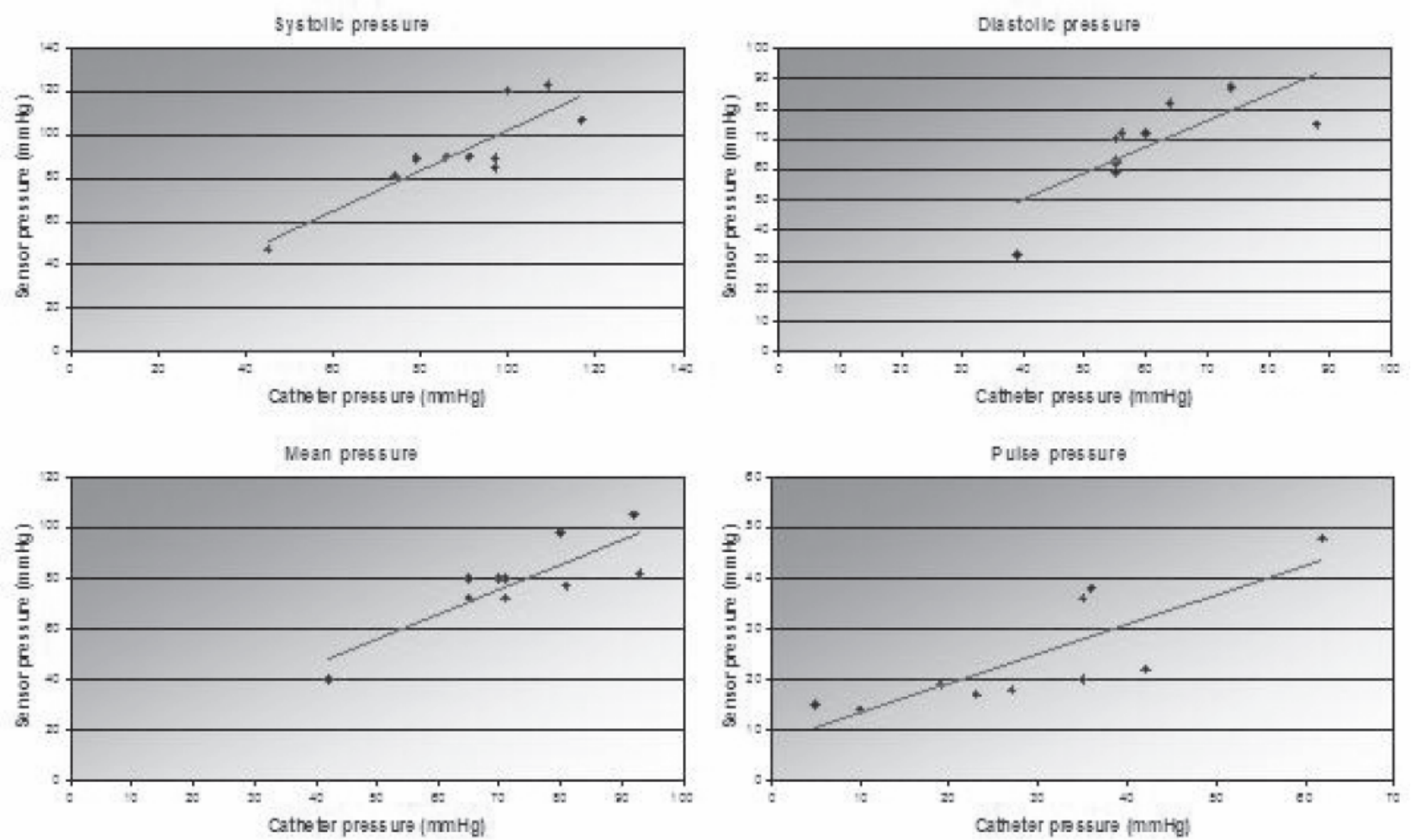

Figure 6 - Scatterplots showing agreement between the Reading 2 pressure measurements of angiographic catheter and pressure sensor. 
Table 4 - Average pressure levels (mmHg) from Readings 1 and 2, and p-values.

\begin{tabular}{|c|c|c|c|c|c|c|c|c|}
\hline \multirow[t]{2}{*}{ Parameter } & \multicolumn{2}{|c|}{ Systolic pressure } & \multicolumn{2}{|c|}{ Diastolic pressure } & \multicolumn{2}{|c|}{ Pulse pressure } & \multicolumn{2}{|c|}{ Mean pressure } \\
\hline & Sensor & Catheter & Sensor & Catheter & Sensor & Catheter & Sensor & Catheter \\
\hline Reading 1 & 112.44 & 117.67 & 62.67 & 59.67 & 49.78 & 57.89 & 81.89 & 80.78 \\
\hline Reading 2 & 90.44 & 86.44 & 68.33 & 60.67 & 22.11 & 25.78 & 78.78 & 72.11 \\
\hline p-value & $>0.05$ & $<0.05$ & $>0.05$ & $>0.05$ & $<0.01$ & $<0.01$ & $>0.05$ & $>0.05$ \\
\hline
\end{tabular}

SOURCE: CardioMEMS Inc., data bank, 2004.

uted to intrasac pressure measurements from patient \#17, for whom a $60 \mathrm{mmHg}$ difference of unidentified origin was recorded. No further comparisons could be made for this patient because the angiographic catheter was removed from the aneurysm sac before Reading 2 pressure gauging was performed. Had the divergent data from this patient not been included, the Pearson's correlation coefficient for the Reading 1 pulse pressure would be 0.71 , with $\mathrm{p}<0.01$.

As mentioned, in four patients, no angiographic catheter readings were obtained, while in nine others catheter readings were obtained solely for Reading 1 . This incomplete collection of data was due to modifications in the sponsor's intraoperative protocol, which underwent a number of alterations implemented over the study's timeframe. As for Reading 2, these changes explain why catheter data was obtained for patient 12 , but not for patients 10 and 11 , as these procedures occurred during a period of debate about whether or not the angiographic catheter was to be used during sensor implantation. Eventually, the use of the angiographic catheter was abandoned altogether during the procedure, with sensor readings compared only to systemic arterial pressure; this analysis goes beyond the scope of this paper, which seeks only to compare methods for direct intrasac pressure measurement.

Owing to the innovative nature of this technology, medical literature is notably scarce on studies investigating the applicability of intrasac pressure sensors. The use of the Remon Medical ImPressure ${ }^{\circledR}$ sensor has been validated by the findings of recent studies. ${ }^{5,10}$ This remote sensor consists of a piezoelectric membrane whose ultrasound-activated capacitor measures the environmental pressure and transmits the values back to the source. In the study by Milner et al. ${ }^{5}$, the pressure sensor was sutured to the suprarenal abdominal aortic wall of six female swines to compare the systolic pressure and pressure waves detected by the sensor and by an angiographic catheter introduced via translumbar percutaneous puncture. Excellent agreement was found between catheter-derived and sensor-derived intrasac pressure measurements, even though no statistical analysis of the data was performed. In the study by Ellozy et al. ${ }^{10}$, the sensor was sutured to the main body of the endoprosthesis (or to its contralateral limb) and implanted in 14 patients. Pressure measurements (systolic, diastolic, and pulse pressure) made with the remote sensor and an angiographic catheter were compared and statistically analyzed by Pearson's correlation. The results obtained demonstrated an excellent correlation that validates the use of the sensor instead of the angiographic catheter. As for the functioning mechanisms, an ultrasound-activated sensor has some limitations depending on the patient's body mass and degree of aortic calcification. ${ }^{10}$ These drawbacks are not applicable for a sensor activated by resonant frequency, which captures the signal even if the antenna is not held against the patient's body.

Despite promising results, the validity of implantable pressure sensors has been debated. The interpretation of sensor-derived pressure readings as the real intrasac pressure assumes that the pressure is evenly distributed within the aneurysm sac. In the case of a mural thrombus, there is evidence that the pressure is consistently higher in the vicinities of the endoprosthesis, although it is minimally elevated when the thrombus is organized. ${ }^{7}$ Furthermore, the difference decreases with time, as the thrombus matures. ${ }^{10}$ Numerous studies have addressed the issue of the influence a thrombus may have on pressure variability within the sac. ${ }^{9,14,15}$ This topic is highly relevant because, in practical terms, a major concern regarding potential differences in sac pressure according to location is that the pressure exerted on the aneurysm wall could be greater than in the lumen; in this case, the sensor reading could underestimate the danger of rupture. However, the intraluminal pressure has been demonstrated to be the same as the aneurysm wall pressure due largely to the permeability of thrombus to fluid, ${ }^{16}$ a factor which also contributes to near equalization of pressure (within 10\%) inside the watertight system created in the aneurysm sac. ${ }^{17}$ In addition, the mentioned studies have all demonstrated that the pressure variability is very small, although the conclusions drawn from these results show some discrepancy. ${ }^{9,14,15}$ It is also important to emphasize that the clinical utility of the sensor is not hindered by these findings, as the pressure changes considered clinically relevant are much greater than the variations seen within the sac, ${ }^{17}$ apart from the fact that the absolute 
pressure measurements are less significant than the trends in intrasac pressure with regard to detecting pressurization due to a possible endoleak. ${ }^{18}$ In the EndoSure ${ }^{\mathrm{TM}}$ study, all sensors were implanted within the aneurysm sac in a position free from existing mural thrombus and, consequently, in direct contact with the environment surrounding the endoprosthesis. Despite these controversies, the validity of a single sensor in determining sac pressure has been echoed by other studies..$^{11,17,18}$

When catheter-derived intra-aneurysm sac pressure measurements were performed by percutaneous translumbar puncture of AAA excluded by endoprosthesis, studies have shown a gradual pressure decrease in the follow-up period. ${ }^{4,7,8}$ This same pattern has been demonstrated in the patients enrolled in the EndoSure ${ }^{\mathrm{TM}}$ study. ${ }^{11}$

Due to the experimental nature of this technology, it is not yet clear what financial burden the use of permanently implantable pressure sensors may bring, even though their long-term costs are likely to be lower than those of other methods. In view of the lack of reported research addressing this particular matter, a more detailed discussion awaits further study.

According to the methodology proposed and based on the findings of this study, it may be concluded that the EndoSure $^{\mathrm{TM}}$ permanently implantable remote pressure sensor produced intrasac pressure readings comparable to those obtained with an angiographic catheter, and is therefore adequate for detecting AAA postexclusion pressurization immediately after endoprosthesis placement. Subsequent data on the EndoSure ${ }^{\mathrm{TM}}$ sensor have been promising, ${ }^{11}$ and long-term follow-up is needed to fully assess its validity for intrasac pressure monitoring.

\section{REFERENCES}

1. Van Marrewijk CJ, Buth J, Harris PL, Norgren L, Nevelsteen A, Wyatt MG. Significance of endoleaks after endovascular repair of abdominal aortic aneurysms: the EUROSTAR experience. J Vasc Surg. 2002;35:461-73

2. Timaran CH, Ohki T, Rhee SJ, Veith FJ, Gargiulo NJ, 3rd, Toriumi H, et al. Predicting aneurysm enlargement in patients with persistent type II endoleaks. J Vasc Surg. 2004;39:1157-62.

3. Wain RA, Marin ML, Ohki T, Sanchez LA, Lyon RT, Rozenblit A, et al. Endoleaks after endovascular graft treatment of aortic aneurysms: classification, risk factors, and outcome. J Vasc Surg. 1998;27:69-80.

4. Dias NV, Ivancev K, Malina M, Resch T, Lindblad B, Sonesson B. Intraaneurysm sac pressure measurements after endovascular aneurysm repair: differences between shrinking, unchanged, and expanding aneurysms with and without endoleaks. J Vasc Surg. 2004;39:1229-35.

5. Milner R, Ruurda JP, Blankensteijn JD. Durability and validity of a remote, miniaturized pressure sensor in an animal model of abdominal aortic aneurysm. J Endovasc Ther. 2004;11:372-7.

6. Pollock JG, Travis SJ, Whitaker SC, Davidson IR, Gregson RH, Hopkinson BR, et al. Endovascular AAA repair: classification of aneurysm sac volumetric change using spiral computed tomographic angiography. J Endovasc Ther. 2002;9:185-93.

7. Sonesson B, Dias N, Malina M, Olofsson P, Griffin D, Lindblad B, et al. Intra-aneurysm pressure measurements in successfully excluded abdominal aortic aneurysm after endovascular repair. J Vasc Surg. 2003;37:733-8.

8. Baum RA, Carpenter JP, Cope C, Golden MA, Velazquez OC, Neschis DG, et al. Aneurysm sac pressure measurements after endovascular repair of abdominal aortic aneurysms. J Vasc Surg. 2001;33:32-41.

9. Vallabhaneni SR, Gilling-Smith GL, Brennan JA, Heyes RR, Hunt JA, How TV, et al. Can intrasac pressure monitoring reliably predict failure of endovascular aneurysm repair? J Endovasc Ther. 2003;10:524-30.
10. Ellozy SH, Carroccio A, Lookstein RA, Minor ME, Sheahan CM, Juta $\mathrm{J}$, et al. First experience in human beings with a permanently implantable intrasac pressure transducer for monitoring endovascular repair of abdominal aortic aneurysms. J Vasc Surg. 2004;40:405-

11. Ohki T, Ouriel K, Silveira PG, Katzen B, White R, Criado F, et al. Initial results of wireless pressure sensing for endovascular aneurysm repair : The APEX Trial - Acute Pressure Measurement to Confirm Aneurysm Sac Exclusion. J Vasc Surg. 2007;45:236-242.

12. Faries PL, Sanchez LA, Marin ML, Parsons RE, Lyon RT, Oliveri S, et al. An experimental model for the acute and chronic evaluation of intraaneurysmal pressure. J Endovasc Surg. 1997;4:290-7.

13. Sanchez LA, Faries PL, Marin ML, Ohki T, Parsons RE, Marty B, et al. Chronic intraaneurismal pressure measurement: an experimental method for evaluating the effectiveness of endovascular aortic aneurysm exclusion. J Vasc Surg. 1997;26:222-30.

14. Xenos ES, Stevens SL, Freeman MB, Pacanowski JP, Cassada DC, Goldman MH. Distribution of sac pressure in an experimental aneurysm model after endovascular repair: the effect of endoleak types I and II. J Endovasc Ther. 2003;10:516-523.

15. Timaran CH, Ohki T, Veith FJ, Lipsitz EC, Gargiulo NJ 3rd, Rhee SJ, et al. Influence of a type II endoleak volume on aneurysm wall pressure and distribution in an experimental model. J Vasc Surg. 2005;41:657663.

16. Adolph R, Vorp DA, Steed DL, Webster MW, Kameneva MV, Watkins SC. Cellular content and permeability of intraluminal thrombus in abdominal aortic aneurysm. J Vasc Surg. 1997;25:916-26.

17. Ohki T. Long-term AAA sac pressure monitoring. Endovascular Today. 2006;5:55-63.

18. Hinnen JW, Koning OH, Visser MJ, Van Bockel HJ. Effect of intraluminal thrombus on pressure transmission in the abdominal aortic aneurysm. J Vasc Surg. 2005;42:1176-1182. 